\title{
Georg Glaeser: Geometry and its Applications - in Arts, Nature and Technology
}

\author{
Springer 2020, $\mathrm{X}+698$ Seiten, ISBN: 978-3-030-61397-6, €58,84; \\ e-Book ISBN 978-3-030-61398-3, €46,00
}

\section{Joachim Hilgert}

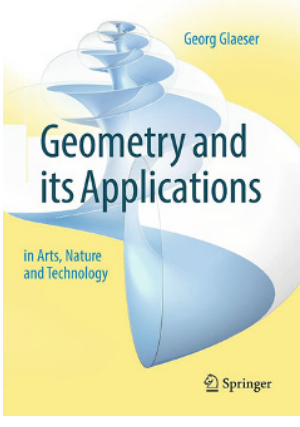

kurzen Essays, die immer wieder um die folgenden thematischen Schwerpunkte kreisen: Die Objekte der euklidischen Geometrie von Punkten und Geraden zu Polyedern, verschiedene Klassen von gekrümmten Flächen im Raum, Licht und Schatten, Perspektive, geometrische Formen in Architektur, Flora und Fauna sowie geometrische Formen in mechanischen Apparaten.

Die Präsentation des Materials ist mehr deskriptiv als deduktiv. Zwar gibt es immer wieder „Sätze“, das heißt Aussagen, die optisch durch eine Box hervorgehoben sind, und dazu auch Beweise. Aber in den meisten Fällen sind die präzisen Voraussetzungen nicht im Einzelnen ausgeführt und die Argumente eher nur skizziert. Besonders auffällig und für den Leser, der versucht die beschriebenen Sachverhalte genau zu verstehen, auch störend ist das fast vollständige Fehlen klarer Definitionen. Als Beispiel seien hier die abwickelbaren Flächen (developable surfaces) genannt.

Joachim Hilgert ( $\square)$

Universität Paderborn, Paderborn, Deutschland

E-Mail: hilgert@upb.de 
Sie kommen im Text über zweihundert mal vor, aber eine als solche gekennzeichnete Definition fehlt. Zwar werden immer wieder Eigenschaften genannt wie ,If this contour only consists of straight lines for every projection, the surface is developable. It is only such surfaces that can be unfolded (developed) into the plane without distortion." Sucht man nach der Definition über den Index, so stellt man fest, dass es dort nur drei Einträge zu ,developable surfaces“ gibt, die im Übrigen alle drei um eine Seite daneben liegen. Es handelt sich also eher nicht um eine „Exampledriven introduction to the broad field of geometry", wie die Internetseite des Verlags zu diesem Buch suggeriert. Es geht dem Autor ganz offensichtlich nicht darum, den Leser in die Lage zu versetzen, selbst abgesichert geometrische Einsichten zu entwickeln.

Was also hat der Autor im Sinn? Seine Darstellung ist mehr impressionistisch, eine Vorstellung vermittelnd, wenn man aus der Distanz schaut, aber nicht mehr greifbar, wenn es ums Detail geht. So gesehen hat der Autor wohl recht, wenn er sagt, es wäre kein spezielles mathematisches Vorwissen für die Lektüre dieses Buches nötig. Die Erklärung für seine Vorgehensweise gibt der Autor mit folgender Aussage auf Seite 261: „However, the issue is very elaborate and does not yield any impressive visuals - it, thus, falls outside the scope of this book."

Damit sind wir bei der eigentlichen Stärke des Buches. Es ist voller wirklich beeindruckender Visualisierungen und auch schöner Aufnahmen geometrischer Objekte. Diese Bilder können Lehrer an Schulen und Hochschulen für ihren GeometrieUnterricht inspirieren und viele Leser würden sich sicher dafür interessieren, wie man sie produziert.

Insgesamt fehlt mir in diesem Buch aber der rote Faden. Es wirkt auf mich zusammengestückelt, was sich auch durch mancherlei Wiederholungen bemerkbar macht, wenn Themen erneut aufgegriffen und ergänzt werden (wie zum Beispiel die abwickelbaren Flächen oder Zentralprojektionen). Auch wirkt die Verbindung von Abbildungen zu erklärendem Text bisweilen lieblos, wenn zum Beispiel in Abschn. 9.2 die Konstruktion der perspektivischen Darstellung eines Hauses erklärt werden soll, die zugehörige Abbildung eine Menge Informationen enthält, die in der Erklärung nicht angesprochen wird, umgekehrt aber nicht alle in der Erklärung benannten Objekte in der Abbildung auftauchen.

Die Lektüre am Stück ist tatsächlich ermüdend und manchmal auch frustrierend, wenn man einen Sachverhalt gerne genau verstehen möchte, die dafür erforderlichen Informationen aber nicht bereit gestellt werden. Manche der Unklarheiten im Text sind allerdings der englischen Übersetzung geschuldet. So lautet etwa der mir unverständliche Satz ,Even the line at infinity of the plane can be determined in this way: It is equal to the center $M$ of the circle“ im Original „Damit lässt sich sogar die Fernebene erfassen: sie entspricht dem Mittelpunkt der Kugel““.

Interessant sind die beschriebenen Querverbindungen und Ähnlichkeiten zu geometrischen Objekten der realen Welt. Leider ist eine zielgerichtete Suche in diesem langen Buch schwierig. Der Index ist ein wenig aleatorisch und hat mir in keinem Fall geholfen, die Begriffserklärungen zu finden, von denen ich angenommen hatte sie nur vergessen zu haben. Überhaupt hat der technische Apparat Luft nach oben. Wiederholt laufen Referenzen ins Leere und erscheinen als [?]. 
„Geometry and its Applications“ ist eine Materialsammlung, in der ich einzelne Stücke sehr gelungener Miniaturen wiedererkannt habe. Es ist voller beeindruckender Visualisierungen. Wenn es aber mehr sein soll als ein „,coffee table book“, sollte man Bilder und Text genauer aufeinander beziehen, es mit einem Glossar und einem zielgerichteten Index ausstatten. Dann hätte der interessierte Leser eine Chance, angedeutete Argumente selbst zu ergänzen.

Funding Open Access funding enabled and organized by Projekt DEAL.

Open Access Dieser Artikel wird unter der Creative Commons Namensnennung 4.0 International Lizenz veröffentlicht, welche die Nutzung, Vervielfältigung, Bearbeitung, Verbreitung und Wiedergabe in jeglichem Medium und Format erlaubt, sofern Sie den/die ursprünglichen Autor(en) und die Quelle ordnungsgemäß nennen, einen Link zur Creative Commons Lizenz beifügen und angeben, ob Änderungen vorgenommen wurden.

Die in diesem Artikel enthaltenen Bilder und sonstiges Drittmaterial unterliegen ebenfalls der genannten Creative Commons Lizenz, sofern sich aus der Abbildungslegende nichts anderes ergibt. Sofern das betreffende Material nicht unter der genannten Creative Commons Lizenz steht und die betreffende Handlung nicht nach gesetzlichen Vorschriften erlaubt ist, ist für die oben aufgeführten Weiterverwendungen des Materials die Einwilligung des jeweiligen Rechteinhabers einzuholen.

Weitere Details zur Lizenz entnehmen Sie bitte der Lizenzinformation auf http://creativecommons.org/ licenses/by/4.0/deed.de. 